\title{
Avaliação ultrassonográfica torácica em cães com efusão pleural e/ou pericárdica
}

\author{
Thoracic ultrasonographyc evaluation of dogs with pleural and/or pericardial effusion
}

\author{
Ana Paula SARRAFF-LOPES ${ }^{1}$; Maria Helena Matiko Akao LARSSON² \\ ${ }^{1}$ Curso de Medicina Veterinária da Pontifícia Universidade Católica do Paraná, São José dos Pinhais - PR, Brasil \\ ${ }^{2}$ Departamento de Clínica Veterinária da Faculdade de Medicina Veterinária e Zootecnia da \\ Universidade de São Paulo, São Paulo - SP, Brasil
}

\begin{abstract}
Resumo
Com o objetivo de avaliar a ultrassonografia torácica (incluindo a ecocardiografia) como método de exame complementar em pacientes portadores de efusão pleural e/ou pericárdica, realizou-se a ultrassonografia torácica em 30 cães, machos e fêmeas de raças e idades variadas. Animais atendidos nos serviços de Pronto-Atendimento, Clínica Médica ou Clínica Cirúrgica do HOVET/USP com efusão pleural e/ou pericárdica, detectadas por meio de exame radiográfico, ultrassonografia emergencial ou toracocentese exploratória foram incluídos no estudo. Observou-se efusão pleural em 12 cães (40\%), efusão pericárdica em oito (26,66\%) e efusão pleural e pericárdica em outros dez cães (33,33\%). A causa da efusão pleural e/ou pericárdica foram avaliadas ultrassonograficamente como sendo: nódulo/tumor cardíaco (5 - 16,66\%), nódulo/tumor intratorácico $(5-16,66 \%)$, insuficiência cardíaca congestiva por cardiomiopatia dilatada $(4-13,33 \%)$ ou endocardiose de mitral e tricúspide (3-10\%), efusão pericárdica idiopática (3 - 10\%), linfossarcoma (2 - 6,66\%), piotórax $(2-6,66 \%)$, ruptura diafragmática $(1-3,33 \%)$, hérnia peritônio-pericárdica $(1-3,33 \%)$, pneumonia e pleurite $(1-3,33 \%)$, tumor de ovário com metástases torácicas ( 1 - 3,33\%), pericardite infecciosa (cinomose) (1 $3,33 \%)$ e hipoalbuminemia $(1-3,33 \%)$. Concluiu-se que a ultrassonografia torácica demonstrou ser um excelente método diagnóstico auxiliar nos casos de efusão pleural e / ou pericárdica, além de ser não invasivo e seguro para o paciente, permitindo guiar punções com segurança e realizar o exame em diferentes decúbitos, evitando assim o estresse do paciente.
\end{abstract}

Palavras-chave: Ultrassonografia torácica. Ecocardiografia. Efusão pleural. Efusão pericárdica. Cães.

\begin{abstract}
To evaluate diagnostic accuracy of thoracic ultrasonography (including echocardiography) of patients with pleural and/or pericardial effusion, thoracic ultrasonography was performed in 30 dogs, males and females, of different breeds and ages. The animals were admitted to the Emergency, Internal medicine or Surgery department of the Faculty of Veterinary Medicine, University of São Paulo, presenting with pleural and/or pericardial effusion, diagnosed by thoracic radiography, emergency thoracic ultrasonography or exploratory thoracocentesis. Twelve (40\%) dogs had pleural effusion, $9(30 \%)$ had pericardial effusion and $9(30 \%)$ had both pleural and pericardial effusions. The definitive cause of effusion were obtained by thoracic ultrasonography as follows: heart mass $(5-16,66 \%)$, intrathoracic mass ( 5 - 16,66\%), congestive heart failure by dilated cardiomyopathy $(4-13,33 \%)$ or mitral and tricuspid insufficiency (3 - 10\%), idiopathic pericardial effusion $(3-10 \%)$, lymphosarcoma $(2-6,66 \%)$, pyothorax $(2-6,66 \%)$, traumatic diaphragmatic hernia $(1-3,33 \%)$, congenital peritoneopericardial hernia $(1-3,33 \%)$, pneumonia and pleuritis $(1$ $-3,33 \%)$, ovarian neoplasia and thoracic metastasis $(1-3,33 \%)$, infectious pericarditis (distemper) $(1-3,33 \%)$ and hypoalbuminemia $(1-3,33 \%)$. The conclusion was that thoracic ultrasonography was an excellent auxiliary exam in animals with pleural and/or pericardial effusion, and it's not invasive and safe for the patient, allowing to guide biopsies and perform the exam in different decubits, avoiding patient stress.
\end{abstract}

Keywords: Thoracic ultrasonography. Echocardiography. Pleural effusion. Pericardial effusion. Dogs.

\section{Introdução}

As aplicações da ultrassonografia torácica no homem e nos pequenos animais são dirigidas, principalmente, para avaliação de doença cardíaca ou do pericárdio ${ }^{1}$. Antigamente, acreditava-se que ela tinha limitada aplicação no diagnóstico das doenças respi-
Correspondência para:

Ana Paula Sarraff-Lopes

Curso de Medicina Veterinária

Pontifícia Universidade Católica do Paraná (PUC-PR)

BR 376, Km 14, São José dos Pinhais, PR - Brasil.

CEP: $83010-500$

e-mail: ana.sarraff@pucpr.br

Recebido: 11/04/2011

Aprovado: 05/10/2011 
ratórias porque o ar dos pulmões impede a transmissão do ultrassom ${ }^{2}$. Entretanto, em doenças em que o pulmão está colapsado, consolidado ou deslocado pela presença de fluido, nódulos ou tumores, a ultrassonografia é viável, revelando informações valiosas que complementam o exame radiográfico ${ }^{1,3,4}$.

A ultrassonografia torácica tem sido indicada para detectar, caracterizar e determinar a etiologia da efusão pleural; doenças mediastinais, pleurais e pulmonares, e para avaliar a integridade do diafragma, sendo um método diagnóstico de inestimável valor para se diferenciar doença cardíaca de não-cardíaca ${ }^{5}$. Além de auxiliar no diagnóstico dessas enfermidades, esse método permite guiar a toracocentese e a biópsia aspirativa por agulha fina ou a biópsia de nódulos/tumores pulmonares, pleurais ou mediastinais, evitando-se assim, estruturas vitais como grandes vasos ${ }^{1,6,7}$.

Sabe-se que, na presença de uma enfermidade torácica como a efusão pleural ou consolidação pulmonar, cria-se uma "janela acústica" no tórax, tornando-se possível o exame sonográfico dessa região ${ }^{6}$. Já pela ecocardiografia, além da detecção de cardiopatias, é possível diagnosticar doenças do pericárdio, bem como lesões nódulo/tumorais cardíacas, as quais comumente causam efusão pericárdica nos cães ${ }^{8,9}$.

As vantagens da ultrassonografia sobre a radiografia torácica são: auxiliar na distinção entre opacidades pulmonares e pleurais e na visibilização de lesões pulmonares periféricas, que estão inaparentes ou obscurecidas ao exame radiográfico e avaliar comorbidades pulmonares, cardíacas e da cavidade pleural. Uma desvantagem associada à ultrassonografia é a inabilidade de se avaliar lesões localizadas abaixo do pulmão normal $^{1,10}$.

Devido a ultrassonografia torácica (incluindo a ecocardiografia) ser de rápida execução, baixo custo e de caráter não invasivo, o presente estudo teve como objetivo avaliar sua sensibilidade como método de exame complementar em pacientes portadores de efusão pleural e/ou pericárdica.

\section{Material e Método}

Foi realizado um estudo observacional, prospectivo, de Coorte, não pareado, não probabilístico com 30 cães, de raças e idades variadas, machos e fêmeas, provenientes do atendimento de rotina do Hospital Veterinário da Faculdade de Medicina Veterinária (HOVET) da Universidade de São Paulo com diagnóstico de efusão pleural e/ou pericárdica.

Como critério de inclusão foi utilizada a presença de efusão pleural e/ou pericárdica, detectadas por meio de exame radiográfico, ultrassonografia emergencial ou toracocentese exploratória.

Após o diagnóstico da efusão, todos os pacientes foram submetidos ao exame ultrassonográfico do tórax e ecocardiografia detalhada. Para tal, os animais foram previamente tricotomizados na região torácica, desde a entrada do tórax até o apêndice xifoide, entre o bordo esternal e a junção costocondral, bilateralmente, podendo se estender até a região dorsal das costelas. Uma camada de gel foi aplicada entre o transdutor e a parede torácica com a finalidade de diminuir a interferência do ar (Carbogel - ULT, Carbogel Ind. e Com. Ltda, São Paulo - SP, Brasil). A escolha da frequência do transdutor baseou-se no porte do animal (EUB$-515^{\mathrm{A}}$, Aparelho de ultrassonografia Hitachi modelo EUB - 515 A, com transdutor bifrequencial, Hitachi). Os animais foram posicionados em decúbito lateral direito e esquerdo e, quando o paciente não tolerava o decúbito lateral devido ao desconforto ou dispneia, o exame era realizado com o animal em estação. $\mathrm{O}$ coração foi avaliado por ecocardiografia bidimensional (modo B), modo M (movimento) e por "Doppler" (pulsado, contínuo e colorido). Os exames foram documentados por meio de impressora (UP 890 - CE, Sony) acoplada ao aparelho. A ecocardiografia foi realizada segundo recomendações da "Echocardiography Committee of the Specialty of Cardiology American College of Veterinary Medicine"11 e autores consultados Henrik ${ }^{12}$; Kienle e Thomas ${ }^{13}$; Boon ${ }^{14}$; 
Kienle $^{15}$ e Moïse e Fox ${ }^{16}$. A ultrassonografia torácica foi realizada de acordo como preconizado pelos autores Stowater e Lamb ${ }^{1}$; Konde e Spaulding ${ }^{17}$, Tidwell ${ }^{6}$; Schwarz e Tidwell ${ }^{18}$ e Matton e Nyland ${ }^{5}$.

Em animais dispneicos, a ultrassonografia torácica foi realizada após a toracocentese, retirando-se somente a quantidade de líquido necessária para aliviar os sintomas clínicos. O exame ultrassonográfico torácico (não cardíaco) foi realizado através dos mesmos espaços intercostais utilizados na ecocardiografia, ou através de outros espaços ou da região subcostal, indicada pelos achados clínicos ${ }^{1}$. O exame radiográfico também auxiliou na escolha da melhor "janela" acústica".

Exames complementares como eletrocardiografia, hemograma, bioquímica sérica, citologia, histopatologia, radiografia e ultrassonografia abdominal foram realizados de acordo com a necessidade dos mesmos, sendo os animais acompanhados até a obtenção do diagnóstico definitivo. Os achados ultrassonográficos foram comparados aos radiográficos, quando realizados, e ao diagnóstico definitivo.

Para as variáveis qualitativas, foram utilizadas as frequências relativas (percentuais) e a frequência absoluta $(\mathrm{N})$ das classes de cada variável. Para as variáveis quantitativas foram utilizadas médias e medianas, para resumir as informações. $\mathrm{Na}$ análise estatística, para as variáveis qualitativas, foi utilizado o teste do Qui-quadrado, para verificar possíveis associações entre as classes das características dos animais. Foi considerado haver associação entre as classes das variáveis quando o resultado do teste apresentou nível de significância menor ou igual a $5 \%$ (valor de $\mathrm{p} \leq 0,05$ ).

\section{Resultados}

Foram incluídos no estudo, em um período de 14 meses, 30 cães com efusão pleural e/ou pericárdica. Destes, 17 (56,66\%) eram machos e 13 (43,33\%), fêmeas.

Quanto à raça, sete $(23,33 \%)$ eram sem raça definida e $23(76,66 \%)$ de raças variadas (Figura 1). A faixa etária variou de um a 13 anos, sendo a mediana igual a nove anos.

Ao exame clínico, as principais alterações dos animais do estudo eram: anorexia, dispneia, cansaço, intolerância ao exercício, perda de peso, ascite, síncope, abafamento de sons cardíacos e/ou respiratórios, taquicardia e pulso fraco.

Observou-se efusão pleural em 22 cães e pericárdica em 18 casos, sendo que 12 apresentaram somente

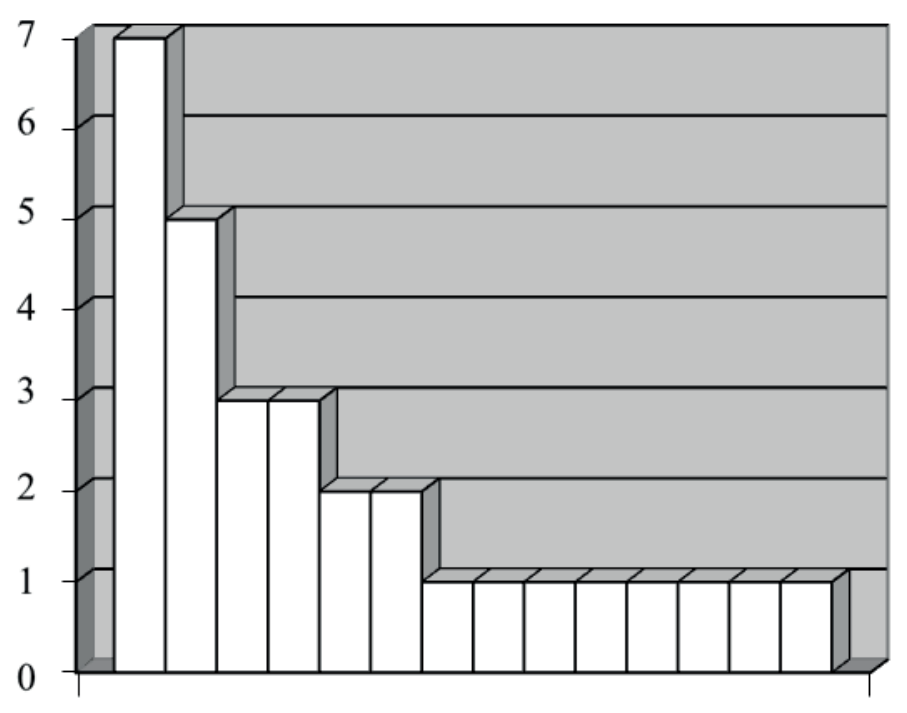

$\square$ SRD
$\square$ Pastor Alemão
$\square$ Poodle
$\square_{\text {Cocker Spaniel }}$
$\square_{\text {Boxer }}$
$\square_{\text {Dogue Alemão }}$
$\square_{\text {Fila Brasileiro }}$
$\square_{\text {Fox Paulistinha }}$
$\square_{\text {Akita }}$
$\square_{\text {Labrador }}$
$\square$ Beagle
$\square$ Fox Terrier
$\square$ Old English Sheepdog
$\square$ Husky Siberiano

Figura 1 - Histograma demonstrando número de cães de cada raça com efusão pleural e/ou pericárdica 
efusão pleural, oito somente pericárdica e dez pleural e pericárdica. As enfermidades diagnosticadas foram (Tabela 1): nódulo/tumor cardíaco (5 - 16,66\%) (Figura 2), nódulo/tumor intratorácico ( 5 - 16,66\%) (Figura 3), insuficiência cardíaca congestiva por cardiomiopatia dilatada ( $4-13,33 \%)$ ou endocardiose de mitral e tricúspide (3 - 10\%) (Figura 4), efusão pericárdica idiopática (3 - 10\%) (Figura 5), linfossarcoma (2-6,66\%) e piotórax $(2-6,66 \%)$. Diagnosticou-se um caso $(1-3,33 \%)$ de: ruptura diafragmática, hérnia peritônio-pericárdica, neoplasia de ovário com metástases torácicas, pneumonia e pleurite, pericardite infecciosa e hipoalbuminemia.

Um grande e variado número de doenças foi identificado nos cães com efusão pleural nesse estudo. A afecção mais comum foi nódulo/tumor intratorácico (cinco casos - massa mediastinal, massa intratorácica, neoplasia de costela, adenocarcinoma pulmonar e sarcoma) seguida por cardiomiopatia dilatada (4) e nódulos/tumores cardíacos (3). O linfossarcoma, pericardite idiopática e piotórax foram as outras causas mais frequentes de efusão pleural (dois casos cada). Detectou-se um caso de: ruptura diafragmática, hérnia peritônio-pericárdica, pneumonia com pleurite e tumor de ovário com metástases torácicas.

A análise e citologia do líquido pleural foram realizadas em 12 cães (40\%), dos quais, um (9,09\%) apresentou transudato simples (nódulo/tumor intratorácico), dois $(18,18 \%)$ transudato modificado (nódulo/ tumor cardíaco; neoplasia de ovário com metástases torácicas), dois (18,18\%) exsudato séptico (piotórax), quatro $(36,36 \%)$ exsudato não séptico (dois animais com linfossarcoma; um com pneumonia e pleurite e um com neoplasia de costela) e três $(27,27 \%)$ efusão hemorrágica (hérnia peritônio pericárdica; pericardite idiopática; nódulo/tumor intratorácico).

As doenças que causaram efusão pericárdica foram: nódulo/tumor cardíaco (cinco casos), seguido de cardiomiopatia dilatada (3), endocardiose de mitral e tricúspide (3) e pericardite idiopática (3).

A análise e citologia do líquido pericárdico foram realizadas em seis casos (20\%), dentre os quais cin-

Tabela 1 - Doenças associadas à efusão pleural e/ou pericárdica em cães

\begin{tabular}{|c|c|c|c|}
\hline Diagnóstico & $\begin{array}{l}\text { Número de } \\
\text { casos }\end{array}$ & Efusão pleural & $\begin{array}{c}\text { Efusão } \\
\text { pericárdica }\end{array}$ \\
\hline \multirow[t]{2}{*}{ Nódulo / tumor cardíaco } & 3 & $\mathrm{X}$ & $\mathrm{X}$ \\
\hline & 2 & & $\mathrm{X}$ \\
\hline Nódulo / tumor intratorácico & 5 & $\mathrm{X}$ & \\
\hline \multirow[t]{2}{*}{ Cardiomiopatia dilatada } & 3 & $\mathrm{X}$ & $\mathrm{X}$ \\
\hline & 1 & $\mathrm{X}$ & \\
\hline Endocardiose mitral e tricúspide & 3 & & $\mathrm{X}$ \\
\hline \multirow[t]{2}{*}{ Efusão pericárdica idiopática } & 2 & $\mathrm{X}$ & $\mathrm{X}$ \\
\hline & 1 & & $\mathrm{X}$ \\
\hline \multirow[t]{2}{*}{ Linfossarcoma } & 1 & $\mathrm{X}$ & $\mathrm{X}$ \\
\hline & 1 & $\mathrm{X}$ & \\
\hline Piotórax & 2 & $\mathrm{X}$ & \\
\hline Pericardite infecciosa & 1 & & $\mathrm{X}$ \\
\hline Hérnia peritônio-pericárdica & 1 & $\mathrm{X}$ & $\mathrm{X}$ \\
\hline Ruptura diafragmática & 1 & $\mathrm{X}$ & \\
\hline Pneumonia e pleurite & 1 & $\mathrm{X}$ & \\
\hline Tumor de ovário com metástases torácicas & 1 & $\mathrm{X}$ & \\
\hline Hipoalbuminemia & 1 & & $\mathrm{X}$ \\
\hline
\end{tabular}




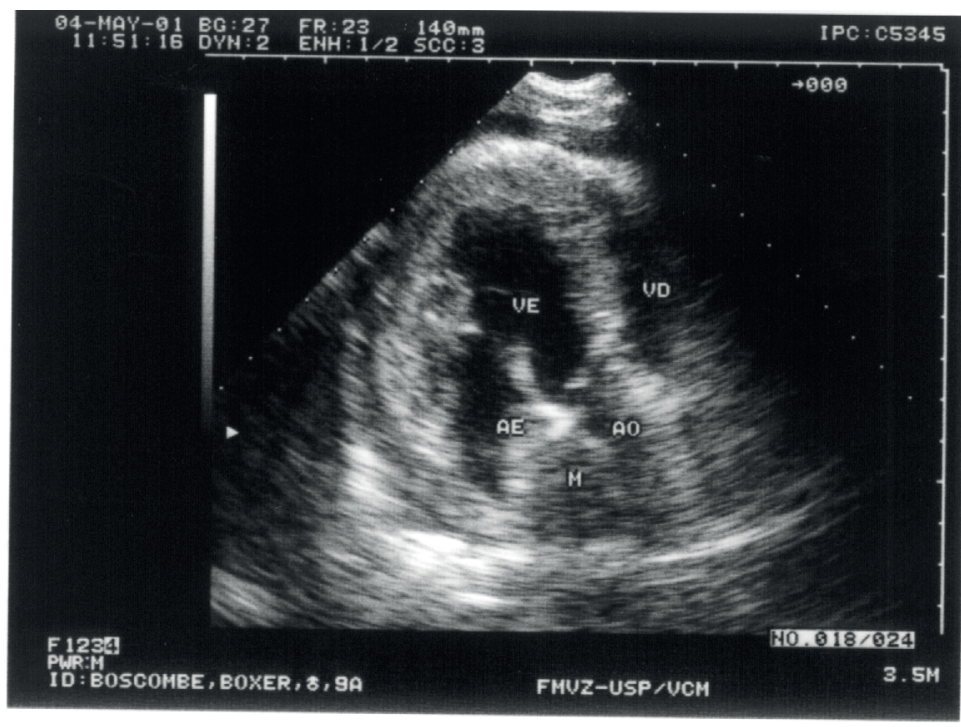

Figura 2 - Imagem ecocardiográfica, obtida na janela para-esternal apical esquerda no corte longitudinal cinco câmaras, demonstrando nódulo/tumor na base do coração e efusão pericárdica em pequena quantidade

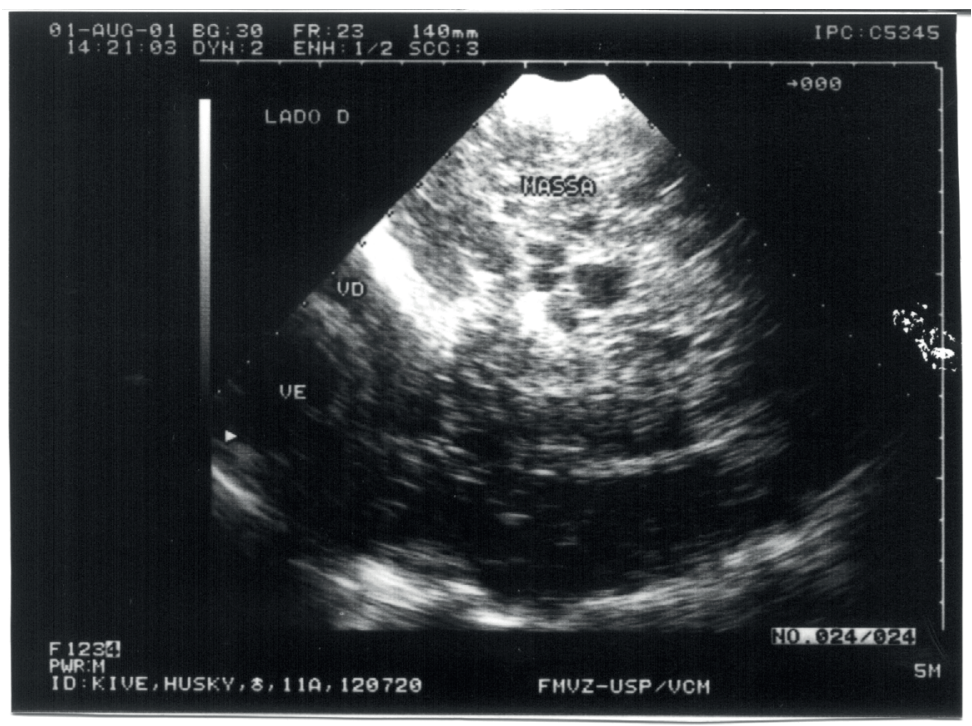

Figura 3 - Imagem ultrassonográfica torácica demonstrando nódulo/ tumor heterogêneo, com múltiplas pequenas áreas císticas adjacente ao coração (Adenocarcinoma pulmonar)

co $(83,33 \%)$ resultaram em efusão hemorrágica (dois com nódulo/tumor cardíaco, dois com pericardite idiopática, um com hérnia peritônio-pericárdica) e um (16,66\%) em transudato simples (hipoalbuminemia).

Nesse estudo, dos 22 animais com efusão pleural, 17 foram submetidos ao exame radiográfico do tórax
(RX), sendo a efusão detectada em 16 casos, não havendo diferença significativa para diagnóstico entre esse método de exame complementar e a ultrassonografia torácica $(\mathrm{p} \geq 0,05)$.

Dos 18 animais com efusão pericárdica, 12 realizaram radiografia torácica e somente em cinco 


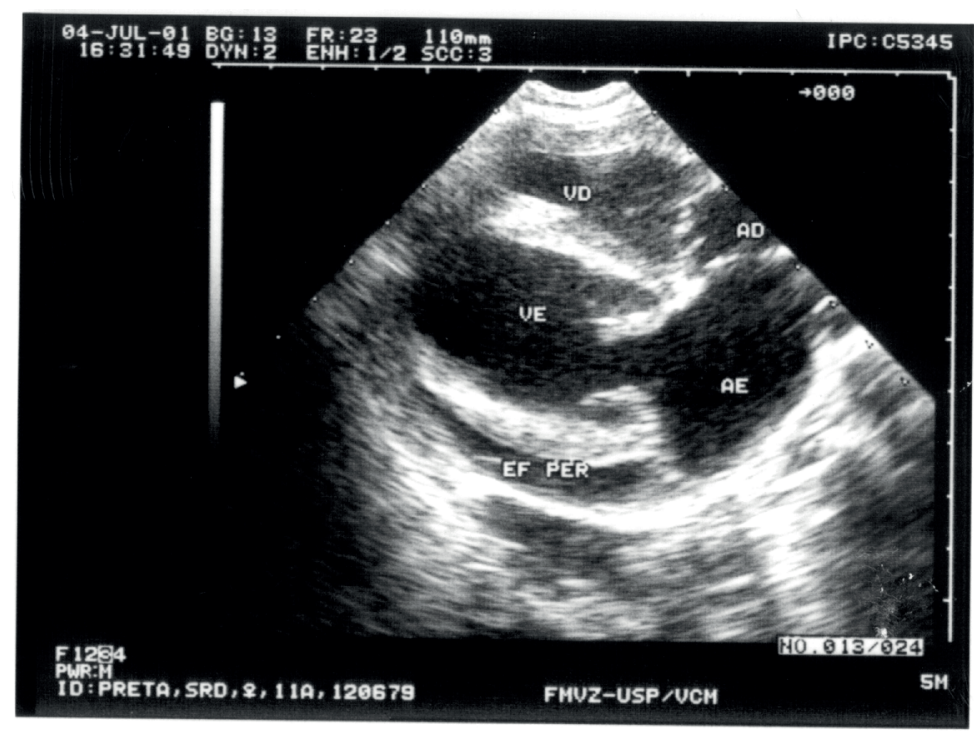

Figura 4 - Imagem ecocardiográfica obtida na janela para-esternal direita, no corte longitudinal quatro câmaras demonstrando espessamento dos folhetos valvares mitral e tricúspide e efusão pericárdica em pequena quantidade (Endocardiose de mitral e tricúspide)

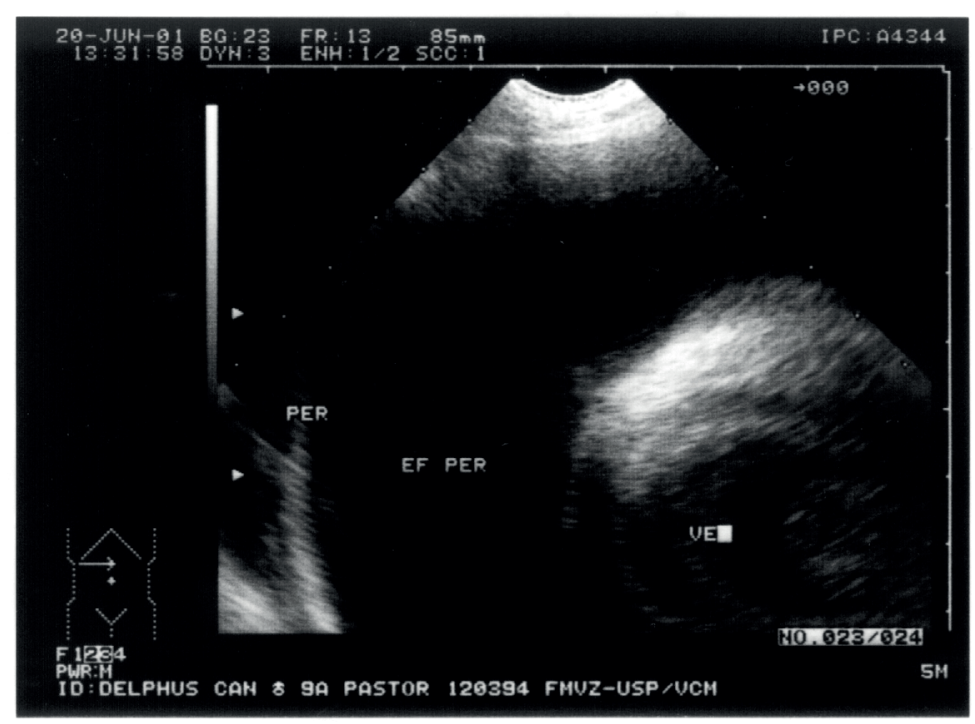

Figura 5 - Imagem ecocardiográfica obtida na janela para-esternal direita no corte transversal do ventrículo esquerdo, demonstrando grande quantidade de efusão pericárdica (Efusão pericárdica idiopática)

casos revelou efusão pericárdica, sendo o exame ultrassonográfico superior para identificação da mesma, em relação ao $\mathrm{RX}(\mathrm{p} \leq 0,05)$. Dos sete $(58,33 \%)$ animais em que o RX foi falso-negativo para efusão pericárdica, a cardiomegalia estava presente em somente um deles (cardiomiopatia dilatada) e a efusão pleural impediu a visualização da silhueta cardíaca em quatro casos. A ecocardiografia permitiu a visibilização da efusão, mesmo em pequenas quantidades. 


\section{Discussão}

Uma discreta predisposição para cães machos (56,66\%) foi observada no presente trabalho, assim como citado na literatura ${ }^{19,20,21}$.

Dentre as raças mais acometidas encontram-se o Pastor Alemão (5 - 16,66\%), Poodle e Cocker Spaniel inglês (três cada - 10\%). No trabalho de Berg e Wingfield ${ }^{19}$ com 42 casos de efusão pericárdica os cães de grande porte predominaram e a raça mais acometida foi o Pastor Alemão (26\%), demonstrando a predisposição dessa raça em apresentar hemangiossarcoma, a neoplasia cardíaca mais frequente. No presente estudo, também se observou prevalência do pastor alemão, porém a doença predominante nessa raça foi a pericardite idiopática (dois casos). Nos estudos de Stafford Johnson et al. ${ }^{21}$ e Mellanby e Herrtage ${ }^{22}$ com efusão pericárdica, a raça mais acometida foi o Golden Retriever, seguida do Pastor Alemão.

Esse estudo ilustra a grande variedade de doenças causadoras de efusão pleural e pericárdica. Dos 22 cães com efusão pleural, a maioria apresentava neoplasia intratorácica $(5-16,66 \%)$, seguido por cardiomiopatia dilatada (quatro casos - 18,19\%), nódulos/ tumores cardíacos (três casos - 13,64\%), linfossarcoma, pericardite idiopática e piotórax (dois casos cada - 9,1\%). Mellanby, Villiers e Herrtage ${ }^{20}$ relataram 81 casos de efusão pleural em cães, com incidência diferente se comparado ao estudo, sendo a afecção mais comum o piotórax (16\%), seguido de pericardite idiopática (8\%), massa mediastinal cranial (7\%), quilotórax idiopático, linfossarcoma, metástases pulmonares e cardiomiopatia dilatada (6\% cada) e nódulos/tumores cardíacos (5\%) entre outras.

Neste estudo, dos 18 casos de efusão pericárdica, as principais afecções foram por nódulos/tumores cardíacos (5 - 27,78\%), cardiomiopatia dilatada, endocardiose de mitral e tricúspide e pericardite idiopática (três cada - 16,67\%), achados esses que estão em consonância com alguns autores ${ }^{19,23,24}$. Em outros estudos, a etiologia prevalente foi a pericardite idiopática, seguida das massas cardíacas e cardiopatias ${ }^{21,22}$.

De 17 animais com efusão pleural (com ou sem efusão pericárdica), o RX não detectou a presença de líquido pleural somente em um caso $(5,89 \%$ de falsos-negativos para a efusão pleural), provavelmente porque a efusão era localizada e em pequeno volume, suportando o achado que, em grandes quantidades de efusão, o exame radiográfico é sensível. Todavia, o estresse do paciente e o desconforto respiratório para o correto posicionamento do animal durante o exame pode ser fatal, ao passo que no exame ultrassonográfico, o animal pode ser avaliado em estação.

Verificou-se nesse estudo que a ultrassonografia é mais eficiente na detecção da efusão pericárdica quando comparada ao exame radiográfico, como também observado por Shaw e Rush ${ }^{25}$. A grande dificuldade na detecção da efusão pericárdica ao exame radiográfico está na diferenciação entre cardiomegalia e efusão em pequena quantidade ${ }^{15}$. A ecocardiografia permitiu não só a visibilização de pequenas quantidades de efusão, bem como os sinais de tamponamento cardíaco, presença de nódulos/tumores (localização, aspecto e tamanho) e alterações secundárias às cardiopatias em todos os casos, como relataram Berg e Wingfield ${ }^{19}$ e Gidlewski e Petrie ${ }^{26}$.

Nos cinco cães com nódulos/tumores intratorácicos (massa mediastinal, massa intratorácica, neoplasia de costela, adenocarcinoma pulmonar e sarcoma), o RX permitiu a visibilização e caracterização da massa, mas em três deles, isso foi possível somente após a toracocentese e drenagem do líquido pleural. A US detectou o nódulo/tumor, sua localização, tamanho, forma, contorno e aspecto, em todos os casos. Esses achados são semelhantes aos descritos por Schwarz e Tidwell ${ }^{18}$, que mencionaram que as principais vantagens da ultrassonografia sobre a radiografia torácica são permitir a distinção entre opacidades pulmonares e pleurais e a detecção de lesões periféricas do parênquima pulmonar, que são 
inaparentes ou estão obscurecidas radiograficamente, pela presença de efusão pleural.

Neste estudo, a avaliação radiológica dos cães com efusão pleural foi importante na avaliação óssea (ex: neoplasia de costela), nos desvios mediastinais que auxiliaram o diagnóstico das massas, no diagnóstico de doenças pulmonares (exemplos: edema pulmonar, metástases pulmonares, opacificações pulmonares) e na orientação para a biópsia e toracocentese. As contribuições da ultrassonografia torácica foram na detecção de nódulos/tumores (localização, tamanho, forma, contorno e aspecto), na observação de efusão pleural e pericárdica (características sonográficas e distribuição), na detecção de alterações no parênquima pulmonar e no auxílio da toracocentese, pericardiocentese e citologia aspirativa.

\section{Conclusões}

Conforme os resultados desse estudo, é possível afirmar que a ultrassonografia é especialmente útil no diagnóstico de doenças pleurais, pericárdicas, cardíacas, mediastinais, afecções na periferia do parênquima pulmonar e lesões diafragmáticas. $\mathrm{O}$ exame ultrassonográfico do tórax é um método não invasivo

\section{Referências}

1. STOWATER, J. L.; LAMB, C. R. Ultrasonography of noncardiac thoracic diseases in small animals. Journal American Veterinary Medical Association, v. 195, n. 4, p. 514-520, 1989.

2.SAITO, T.; KOBAYASHI, H.; KITAMURA, S. Ultrasonographic approach to diagnosing chest wall tumors. Chest, v. 94, n. 6, p. 1271-1274, 1988.

3. WEINBERG, B.; DIAKOUMAKIS, E. E.; KASS, E. G.; SEIFE, B.; ZVI, Z. B. The air bronchogram: sonographic demonstration. American Journal of Roentgenology, v. 147, n. 3, p. 593-595, 1986.

4.LARSON, M. M. Ultrasound of the Thorax (Noncardiac). Veterinary Clinics of North America. Small Animal Practice, v. 39, n. 4, p. 733-745, 2009.

5. MATTON, J. S.; NYLAND, T. G. Thorax. In: NYLAND, G. T.; MATTON, J. S. Small Animal diagnostic ultrasound. 2nd ed. Philadelphia: W.B. Saunders, 2002. p. 325-353.

6. TIDWELL, A. S. Ultrasonography of the thorax (excluding the heart). Veterinary Clinics of North America. Small Animal Practice, v. 28, n. 4, p. 993-1015, 1998. e seguro para o paciente e permite guiar punções com acurácia e realizar o exame em diferentes decúbitos, evitando assim o estresse do paciente.

Neste estudo, o exame radiográfico dos cães com efusão pleural mostrou-se importante na avaliação óssea (ex: neoplasia de costela), nos desvios mediastinais que facilitaram o diagnóstico dos nódulos/tumores, no diagnóstico de doenças pulmonares (edema pulmonar, metástases pulmonares, opacificações pulmonares) e na orientação para a biópsia e toracocentese, enquanto que as contribuições da ultrassonografia torácica foram: detecção de nódulos/tumores (localização, tamanho, forma, contorno e aspecto), observação de efusão pleural e pericárdica (características sonográficas e distribuição), detecção de alterações no parênquima pulmonar e auxiliar na toracocentese, pericardiocentese e citologia aspirativa.

Apesar das inúmeras vantagens, a ultrassonografia torácica tem como limitações: adequada janela acústica requerida e a não propagação da onda sonora através das interfaces ar-tecido, impossibilitando assim, a visibilização de lesões no pulmão aerado. Seus achados e sua interpretação também dependem da habilidade e experiência do executor do exame.

7.REICHLE, J. K.; WISNER, E. R. Non-cardiac thoracic ultrasound in 75 feline and canine patients. Veterinary Radiology and Ultrasound, v. 41, n. 2, p. 154-162, 2000.

8. THOMAS, W. P.; SISSON, D.; BAUER, T. G.; REED, J. R. Detection of cardiac masses in dogs by two-dimensional echocardiography. Veterinary Radiology, v. 25, n. 2, p. 65-72, 1984.

9. SISSON, D.; THOMAS, W. P. Pericardial disease and cardiac tumors. In: FOX, P. R.; SISSON, D.; MOÏSE, N. S. Textbook of canine and feline cardiology. Philadelphia: W.B. Saunders, 1999. p. 679-701.

10.TIDWELL, A. S. Diagnostic pulmonary imaging. Problems in Veterinary Medicine, v. 4, n. 2, p. 239-264, 1992.

11.LOMBARD, C. V. Normal values of the canine M-mode echocardiogram. American Journal Veterinary Research, v. 45, n. 10, p. 2015-2018, 1984.

12.HENRIK, R. A. Echocardiography and doppler ultrasound. In: MILLER, M. S.; TILLEY, L. P. Manual of canine and feline cardiology. 2nd ed. Philadelphia: W.B. Saunders, 1995. p. 75-107. 
13. KIENLE, R. D.; THOMAS, W. P. Echocardiography. In: NYLAND, T.; MATOON, J. S. Veterinary diagnostic ultrasound. Philadelphia: W.B. Saunders, 1995. p. 198-225

14.BOON, J. A. Manual of veterinary echocardiography. Baltimore: Williams and Wilkins, 1998. 478 p.

15. KIENLE, R. D. Pericardial disease and cardiac neoplasia. In: KITTLESON, M. D.; KIENLE, R. D. Small animal cardiovascular medicine. St. Louis: Mosby, 1998. p. 413-432.

16. MOÏSE, N. S.; FOX, P. R. Echocardiography and Doppler imaging. In: FOX, P. R.; SISSON, D.; MOÏSE, N. S. Textbook of canine and feline cardiology. Philadelphia: W.B. Saunders, 1999. p. 130-171.

17.KONDE, L. J.; SPAULDING, K. Sonographic evaluation of the cranial mediastinum in small animals. Veterinary Radiology, v. 32 , n. 4 , p. $178-184,1991$.

18. SCHWARZ, L. A.; TIDWELL, A. S. Alternative imaging of the lung. Clinical Techniques in Small Animal Practice, v. 14, n. 4, p. 187-206, 1999.

19.BERG, R. J.; WINGFIELD, W. E. Pericardial effusion in the dog: a review of 42 cases. Journal American Animal Hospital Association, v. 20, n. 5, p. 721-730, 1984.

20. MELLANBY, R. J.; VILLIERS, E.; HERRTAGE, M. E. Canine pleural and mediastinal effusions: a retrospective study of 81 cases. Journal Small Animal Practice, v. 43, n. 10, p. 447-451, 2002.

21.STAFFORD JOHNSON, M.; MARTIN, M.; BINNS, S.; DAY M. J. A retrospective study of clinical findings, treatment and outcome in 143 dogs with pericardial effusion. Journal Small Animal Practice, v. 45, n. 11, p. 546-552, 2004.

22. MELLANBY, R. J.; HERRTAGE, M. E. Long-term survival of 23 dogs with pericardial effusions. Veterinary Record, v. 156, n. 18, p. 568-571, 2005.

23.SHAW, S. P.; RUSH, J. E. Canine pericardial effusion: pathophysiology and cause. Compendium on Continuing Education for the Practicing Veterinarian, v. 29, n. 7, p. 400404, 2007a.

24.NELSON, O. L.; WARE, A. W. Pericardial effusion. In BONAGURA, J. D.; TWEDT, D. C. (Ed.). Kirk's current veterinary therapy XIV small animal practice. Philadelphia: W.B. Saunders, 2009. p. 825-831.

25.SHAW, S. P.; RUSH, J. E. Canine pericardial effusion: diagnosis, treatment, and prognosis. Continuing Education for the Practicing Veterinarian, v. 29, n. 7, p. 405-411, $2007 \mathrm{~b}$.

26. GIDLEWSKI, J.; PETRIE, J. Pericardiocentesis and principles of echocardiographic imaging in the patient with cardiac neoplasia. Clinical Techniques is Small Animal Practice, v. 18, n. 2, p. 131-134, 2003. 